\title{
A failed case of acupuncture therapy for malignant bowel obstruction
}

\author{
Sinan Tian', Yingfan Chen ${ }^{1}$, Jifu $\mathrm{E}^{2}$ and Shi Shu ${ }^{1 *}$ \\ ${ }^{1}$ Changhai Hospital of Traditional Chinese Medicine, Second Military Medical University; 168Changhai Road, Yangpu District, Shanghai, China \\ ${ }^{2}$ Department of Colorectal Surgery, Changhai Hospital, Second Military Medical University, 168Changhai Road, Yangpu District, Shanghai 200433, China
}

\begin{abstract}
Malignant bowel obstruction (MBO) is a frequent complication in advanced cancer patients, especially in those with abdominal tumors. Acupuncture is considered effective worldwide in the treatment of $\mathrm{MBO}$ in recent clinical research, and has been used for thousands of years in China. But here is a failed acupuncture treatment with conventional treatment for MBO. It was found that not all bowel obstructions are suitable for acupuncture treatment. Meanwhile, the assessment of patient's physical condition is often overlooked before acupuncture treatment is selected, because the adverse events of acupuncture are considered to be infrequent and mild. These neglects are often fatal for patients. We hope that this failed case can provide a reference for clinicians, so as to enable patients to receive greater benefits in the future.

Key clinical message: Malignant bowel obstruction (MBO) is a frequent complication in advanced cancer patients. Alternative therapy combined with conventional treatment might benefit patients. However, there remains a great number of uncertainties about the efficacy of alternative therapy. The patient's physical condition and etiology of bowel obstruction must be considered fully if acupuncture treatment is selected. Especially, patients with advanced cancer should be promptly and cautiously treated.

A failed case of acupuncture therapy for malignant bowel obstruction: Malignant bowel obstruction (MBO) is a frequent complication in advanced cancer patients, especially in those with abdominal tumors. Alternative therapy combined with conventional treatment might benefit patients. However, there remains a great number of uncertainties about the efficacy of alternative therapy. Herein, a failed case of acupuncture therapy for $\mathrm{MBO}$ will be reported, and the authors received consent for publication from the patient.
\end{abstract}

\section{Case report}

A 62-year-old gentleman, whose chief complaint was intense nausea and vomiting for almost one month, was hospitalized for gastric cancer. During the surgery, there were $100 \mathrm{ml}$ turbid ascites in the abdominal cavity. The tumor was located in the greater curvature side of the gastric antrum and penetrated the serosal layer. Besides, several obviously enlarged lymph nodes were found around the stomach. Additionally, a number of hard lumps, some of which even coalesced, were observed in the peritoneum and mesentery of small intestine and colon. The patient got a palliative surgery of gastrojejunostomy to reestablish the digestive permeability, instead of radical surgery, because the tumor had extensive intra-abdominal metastasis. Pathological results showed gastric adenocarcinoma, poorly differentiated omental adenocarcinoma and signet-ring cell carcinoma.

After the surgery, the patient complained about abdominal pain, abdominal distension and nausea. Meanwhile, he couldn't pass gas or defecate without paraffin oil enema after gastrointestinal decompression tube was pulled out. The number of bowel sounds reduced to less than once per minute. Straight abdominal X-ray position did not show clear gas-liquid plane. Considering the oppression of metastatic tumor and more than $1500 \mathrm{ml}$ digestive juice every day, the patient was diagnosed as $\mathrm{MBO}$. With regard to plenty of ascites and abdominal metastatic tumor, the patient was treated with nasojejunal decompression and correction of the fluid and electrolyte imbalance, rather than surgery for MBO. However, all the attempts to improve $\mathrm{MBO}$ were unsuccessful. On the basis of conventional therapy, acupuncture treatment was added.
We undertook a course (4 days) of acupuncture treatment every morning. The needles (diameter, $0.3 \mathrm{~mm}$; length, $40 \mathrm{~mm}$ ) were inserted 3-5 mm into ST36, ST37, ST39 and LR3, lifted, thrust, twisted and twirled by hand every $5 \mathrm{~min}$, and left in place for $30 \mathrm{~min}$ once a day. During the treatment of the first two days, the patient did not feel any discomfort. While fatigue immediately appeared the next day, but it disappeared after a nap. After the fourth acupuncture treatment, the symptom of fatigue became even worse. The patient wasn't able to walk by himself till the afternoon. During the period of treatment, the abdominal pain with distention hadn't been relieved, and the number of bowel sounds also did not increase. Consequently, acupuncture treatment was stopped. The patient still needed paraffin oil enema to pass stool until he was discharged. The patient was said to die of $\mathrm{MBO}$ two months later through telephone follow-up.

\section{Discussion}

The dual regulatory effects of traditional treatment make it possible to promote bowel peristalsis in any stage of cancer treatment while there are no other treatment options [1]. Acupuncture has been used worldwide as an appropriate treatment for bowel obstruction. As shown

Correspondence to: Shi Shu, Changhai Hospital of Traditional Chinese Medicine, Second Military Medical University; 168Changhai Road, Yangpu District, Shanghai-200433, China, Tel: +86 13761312876; E-mail: shushitcm@163.com

Received: June 04, 2017; Accepted: June 22, 2017; Published: June 24, 2017 
by the experiments, the acupuncture points chosen can effectively treat $\mathrm{MBO}$ [2] and the adverse events of acupuncture are infrequent and mild [3]. Unfortunately, this was a failed case by acupuncture therapy for MBO. Meanwhile, acupuncture might increase the chance of fatigue. The possible reasons might be ignored in the treatment period. Firstly, it is very important to consider the etiology of MBO. Mechanical bowel obstruction cannot be relieved by acupuncture [4]. Cancerous etiology of $\mathrm{MBO}$ also needs to be carefully and seriously considered. Secondly, patients' tolerance to acupuncture is a significant evaluation index. As shown by this case, the MBO patient with poor physical condition is not recommended to receive acupuncture which may aggravate cancer-related fatigue. It is difficult to choose acupuncture treatment correctly because the clinical diagnosis and etiology of MBO are often unclear in the early stage. In the paper about the management of patients with $\mathrm{MBO}$ in US, the management rarely requires an acute and long-term decision [5]. Hence, it is suggested that acupuncture can be selected for MBO if patients are willing to accept this traditional treatment and convey the dissatisfaction with conventional treatment. Before the acupuncture treatment, the physical condition must be quantified assessment by using ECOG score standard and Karnofsky score standard. If the symptoms of $\mathrm{MBO}$ have not been improved within 24-48 hours, acupuncture is considered being unable to relieve $\mathrm{MBO}$ and it should be abandoned immediately.

\section{Conclusion}

Clinically, many patients with $\mathrm{MBO}$ encountered the same problem. Because of poor physical condition, some patients often cannot tolerate surgery or are unable to obtain benefit from surgery which can increase the incidence of the secondary MBO. These patients who loss surgery opportunities will receive the palliative and alternative treatment. The patient's physical condition and etiology of bowel obstruction must be considered fully if acupuncture treatment is selected. Especially, patients with advanced cancer should be promptly and cautiously treated. We hope that this failed case can provide a reference for clinicians, so as to enable patients to receive greater benefits in the future.

\section{References}

1. Ling CQ, Yue XQ, Ling C (2014) Three advantages of using traditional Chinese medicine to prevent and treat tumor. J Integr Med 12: 331-335. [Crossref]

2. Ng SS, Leung WW, Mak TW, Hon SS, Li JC, et al. (2013) Electroacupuncture reduces duration of postoperative ileus after laparoscopic surgery for colorectal cancer. Gastroenterology 144: 307-313. [Crossref]

3. Lau CH, Wu X, Chung VC, Liu X, Hui EP, et al. (2016) Acupuncture and related therapies for symptom management in palliative cancer care: systematic review and meta-analysis. Medicine (Baltimore) 95: e2901. [Crossref]

4. Bi HJ, He YY, Shu S, Zhou QH (2013) Failed acupuncture treatment of small intestinal obstruction after distal gastric cancer: a case report. Acupunct Med 31: 334-335. [Crossref]

5. Alese OB, Kim S, Chen Z, Owonikoko TK, El-Rayes BF (2015) Management patterns and predictors of mortality among US patients with cancer hospitalized for malignan bowel obstruction. Cancer 121: 1772-1778. [Crossref]

Copyright: (C2017 Tian S. This is an open-access article distributed under the terms of the Creative Commons Attribution License, which permits unrestricted use, distribution, and reproduction in any medium, provided the original author and source are credited. 